Ester de S. Costa 1

Ione Morita 2

Miguel A. R. Martinez ${ }^{2}$

\section{Percepção dos efeitos do trabalho em turnos sobre a saúde e a vida social em funcionários da enfermagem em um hospital universitário do Estado de São Paulo}

\author{
Nursing staff perceptions of the effects \\ of shift work on health and social life \\ at the São Paulo State University hospital
}

1 Faculdade de Medicina de Botucatu, Universidade Estadual Paulista. C. P. 549, Botucatu, SP 18618-970, Brasil. 2 Departamento de Saúde Pública, Faculdade de Medicina de Botucatu, Universidade Estadual Paulista. Botucatu, SP 18618-970, Brasil.

\begin{abstract}
There is a relationship between shift work and the beginning of organized life. Health services require shift work to keep activities running twenty-four hours a day. This study thus aimed to identify nursing staff shift work systems in a university hospital, evaluate health workers' perceptions of the possible effects of shift work on their health and social life, and assess workers' participation in preparing nursing schedules. In terms of materials and methods, this was an exploratory and descriptive study with a sample of 348 nursing staff members, using an appropriate questionnaire. Most were married women under 40 with at least one child, working on rotating shifts with more than fifteen-days frequency of rotation, and with neurological, psychological, gastrointestinal, and cardiovascular health complaints. They also reported relational problems on the job. Most of the nursing staff played a minor role in preparing nursing schedules. Key words Shift Work; Nursing Team; Occupational Health
\end{abstract}

Resumo O trabalho em turnos existe desde o início da vida social dos homens, sendo utilizado em diferentes setores, como na indústria de produção de bens de consumo e de serviços. A área da saúde exige o sistema em turnos para manutenção de atividades durante 24 horas. Teve-se como objetivo identificar os sistemas de turnos em funcionários de enfermagem em um hospital universitário, avaliar a percepção sobre os prováveis efeitos do trabalho em turnos em sua saúde e vida social e o grau de participação desses funcionários na forma de organização de sua jornada. Trata-se de uma pesquisa descritiva e exploratória, cujos resultados foram obtidos mediante questionário aplicado a 348 funcionários da enfermagem do Hospital de Clínicas da Faculdade de Medicina de Botucatu, São Paulo. Dentre os resultados, observou-se que, em sua maioria, eles tinham menos de 40 anos, eram casados, do sexo feminino, com um filho pelo menos, cumprindo uma escala de turnos alternados, com freqüência de revezamento superior a quinze dias e referindo queixas de caráter neuro-psíquico, gastrintestinal e cardiovascular. Houve ainda queixas sobre relacionamento e tempo de convivência. Na maioria das vezes, era pouca a participação do funcionário na forma de organização de sua escala de trabalho.

Palavras-chave Trabalho em Turnos; Equipe de Enfermagem; Saúde Ocupacional 


\section{Introdução}

O trabalho em turnos existe desde o início da vida social dos homens e, atualmente, além de razões tecnológicas e econômicas, os turnos estão sendo introduzidos, de forma crescente, em função do atendimento à população. A área da saúde é um setor que utiliza as escalas em turnos, dada a necessidade de manutenção das atividades durante as 24 horas, tendo um contingente expressivo de trabalhadores (Rutenfranz et al., 1989; Triolo, 1989; Paim, 1994).

Em decorrência do trabalho em turnos poderão ocorrer alterações de sono, distúrbios gastrintestinais, cardiovasculares e desordens psíquicas e, em relação à vida social e à família, ressalta-se o prejuízo na participação de atividades sociais organizadas, como as escolares, culturais, esportivas, etc., dificultando sua integração na vida social da comunidade (Åkerstedt, 1988; Vener et al., 1989).

Tendo em vista a problemática que o trabalho em turnos acarreta, os objetivos da presente pesquisa foram identificar as características organizacionais dos sistemas de turnos existentes na área de enfermagem de um hospital universitário do Estado de São Paulo, descrever a percepção dos possíveis efeitos do trabalho em turnos na saúde e na vida social dos funcionários do corpo de enfermagem e avaliar o seu grau de participação na elaboração de suas próprias escalas de trabalho.

\section{Metodologia}

O trabalho foi desenvolvido no Hospital das Clínicas (fundado em 1967) da Faculdade de Medicina de Botucatu, Universidade Estadual Paulista Júlio de Mesquita Filho.

Para se chegar ao universo de entrevistados, excluíram-se do total (791 funcionários) os funcionários em licença, em férias e os afastados; os que atuavam em ambulatórios, hemoterapia, hospital-dia e área administrativa, portanto, consideraram-se apenas os que estavam em unidade de internação (348). Entre estes últimos, separaram-se dois grupos: os que tinham turno permanente e os de turno alternado (278), mas não foi objetivo compará-los, assim como não se analisou a competência de cada função.

Aplicou-se um questionário com perguntas abertas e fechadas, com as seguintes variáveis: função, idade, sexo, estado civil e número de filhos; as dificuldades que o trabalho em turno poderia acarretar, relacionadas ao ritmo circadiano individual; a escala de trabalho e tópicos relacionados à organização das escalas de trabalho.

\section{Resultados}

Com relação à categoria profissional, observou-se o predomínio do auxiliar $(64,65 \%)$ e do atendente de enfermagem $(23,27 \%)$, apenas $5,45 \%$ tinham nível universitário, 2,30\% eram técnicos de enfermagem e 4,31\%, auxiliar de serviços de saúde. Quanto ao sexo, 80,75\% eram femininos e $19,25 \%$, masculinos, sendo a maioria casada $(47,98 \%)$, seguindo-se os solteiros $(30,74 \%)$ e desquitados $(11,20 \%)$.

A faixa etária predominante era de 25-30 anos $(23,56 \%)$, seguindo-se a de $30-35$ anos (20,68\%), a de 35-40 anos (19,82\%), e a de $40-45$ anos (14,08\%). Abaixo de dez por cento, é interessante observar a presença de um contingente jovem, de 20 a 25 anos (8,04\%), com valores similares ao de um mais velho, de 45 a 50 anos $(7,75 \%)$.

A maioria dos funcionários $(24,71 \%)$ possuía dois filhos; 22,98\%, um filho; seguindo-se-lhe nenhum filho $(20,40 \%)$ e, três filhos $(16,66 \%)$. O número máximo de filhos mencionado foi nove.

O sistema de turnos alternados era predominante $(79,88 \%)$ sobre o permanente $(18,67 \%)$. Considerando-se apenas o grupo de 278 funcionários em sistemas de turnos alternados, observou-se que a estrutura circadiana da maioria era matutina $(32,73 \%)$, seguindo-se os vespertinos $(31,65 \%)$, os indiferentes $(18,34 \%)$ e os intermediários $(17,26 \%)$ (Tabela 1). Dentre esses 278 profissionais, $102(36,69 \%)$ referiram que os critérios para o estabelecimento dos dias de folga, incluindo-se sua distribuição durante a semana, foram decididos pela chefia e definidos em escalas, seguidos pelos que, além da escala, tinham como critério as questões dos feriados e finais de semana $(33,09 \%)$, e, em percentual menor, $16,18 \%$ tiveram os seguintes critérios: escala, feriados, fins de semana e necessidade pessoal.

Em um período de 30 dias, a maioria dos funcionários $(45,68 \%)$ teve, em média, oito dias de folga e $25,00 \%$, entre oito e dez dias de folga e 7,18\%, 10 dias de folga. Relacionando dias de folga e fins de semana, verificou-se que a maioria $(47,41 \%$ e $34,48 \%)$ teve um ou dois dias de folga, respectivamente, coincidindo com fins de semana e $10,91 \%$ tiveram quatro dias de folga coincidentes com finais de semana.

Cento e dezessete funcionários $(42,09 \%)$ cumpriam uma escala de turno com freqüência de revezamento superior a 15 dias, e noventa e três $(33,45 \%)$, uma escala com freqüência de revezamento semanal (Tabela 2).

De acordo com sua percepção, o desempenho físico, os distúrbios mentais, neurológicos e psiquiátricos foram os aspectos da saúde mais citados pelos funcionários em sistemas alternados, seguidos de distúrbios gastrintestinais. 
A interferência no relacionamento pessoal/ familiar foi o efeito mais citado do trabalho em turnos, seguido por restrições de atividades sociais e dificuldade de planejamento da vida.

\section{Conclusões}

Considerando-se a composição da equipe de enfermagem, é muito baixo o número de profissionais de nível superior, especialmente para um hospital terciário. Ocorre, também, uma divisão técnica do trabalho que opera verticalmente, com base na redistribuição das tarefas entre pessoas de diferentes níveis de qualificação, que gera uma importante demanda de pessoal auxiliar, por ser menos custosa. Esta distribuição reafirma uma situação não compatível com o porte de um hospital universitário.

O predomínio do sexo feminino nesta amostra está de acordo com os dados observados na literatura, confirmando-se uma tendência à "feminilização" da força de trabalho em saúde. Verifica-se, também, a predominância de um pessoal em plena capacidade produtiva, em sua maioria casado e com filhos, indicando que suas vidas familiar e pessoal sofrem restrições em decorrência do esquema de trabalho em turnos. Os solteiros estão sujeitos a outras restrições na esfera social, cuja intensidade irá depender da forma de organização de sua jornada de trabalho, ou seja, das escalas de trabalho.

O sistema de turno alternado era cumprido pela maioria. De acordo com sua percepção sobre os efeitos do trabalho em turnos, a saúde era afetada principalmente por distúrbios neuro-psíquicos, cardiovasculares e gastrintestinais, acompanhando os dados de literatura.

Da mesma forma, a vida social era prejudicada, em primeiro lugar, pela interferência no relacionamento pessoal e familiar; em segundo, pelas restrições das atividades sociais e, em terceiro, pela dificuldade de planejar a vida.

Os sistemas de turnos identificados foram o permanente e o alternado, com predomínio deste, apresentando uma freqüência de revezamento superior a quinze dias.

A participação dos funcionários na forma de organização de sua jornada de trabalho e no estabelecimento dos dias de folga foi mínima, ficando a elaboração das escalas a cargo das chefias. Fundamentando-se na identificação destes problemas, sugeriu-se o retorno da análise ao pessoal envolvido e, conjuntamente, com as chefias, procurar estratégias e mecanismos para superá-los e, em caso de impossibilidade prática, minimizá-los.
Tabela 1

Distribuição dos funcionários de enfermagem,

em sistema de turnos alternados, segundo estrutura circadiana, no Hospital de Clínicas da Faculdade de Medicina da Universidade Estadual Paulista, 1995.

\begin{tabular}{lcc}
\hline Indivíduos & $\mathbf{n}$ & $\%$ \\
\hline Matutinos & 91 & 32,73 \\
Vespertinos & 88 & 31,65 \\
Intermediários & 48 & 17,26 \\
Indiferentes & 51 & 18,34 \\
Sem referência & - & - \\
Total & 278 & 100,00 \\
\hline
\end{tabular}

Tabela 2

Distribuição dos funcionários de enfermagem, em sistemas alternados, segundo freqüência de revezamento, no Hospital de Clínicas da Faculdade de Medicina da Universidade Estadual Paulista, 1995.

\begin{tabular}{lrr}
\hline $\begin{array}{l}\text { Freqüência } \\
\text { de revezamento }\end{array}$ & $\mathbf{n}$ & \multicolumn{1}{c}{$\%$} \\
\hline 02 & 19 & 6,83 \\
07 & 93 & 33,45 \\
15 & 9 & 3,24 \\
+15 & 117 & 42,09 \\
Outra & 3 & 1,08 \\
Sem informação & 37 & 13,31 \\
Total & 278 & 100,00 \\
\hline
\end{tabular}

\section{Referências}

ÅKERSTEDT, T., 1988. Sleepiness as a consequence of shift work. Sleep, 11:17-34.

PAIM, J. S., 1994. Recursos Humanos em Saúde no Brasil. Problemas Crônicos e Desafios Agudos. São Paulo: Faculdade de Saúde Pública, Universidade de São Paulo.

RUTENFRANZ, J.; KNAUTH, P. \& FISCHER, F. M., 1989. Trabalho em Turnos e Noturno. São Paulo: Editora Hucitec.

TRIOLO, P. K., 1989. Occupational health hazards of hospital staff nurses. American Association of Occupational Health Nurses Journal, 37:232-237.

VENER, K. J.; SZABO, S. \& MOORE, J. G., 1989. The effect of shift work on gastrointestinal (GI) function: A review. Chronobiologia, 16:421-439. 